\title{
Multichannel Blind Deconvolution of Spatially Misaligned Images
}

\author{
Filip Šroubek and Jan Flusser, Senior Member, IEEE
}

\begin{abstract}
Existing multichannel blind restoration techniques assume perfect spatial alignment of channels, correct estimation of blur size, and are prone to noise. We developed an alternating minimization scheme based on a maximum a posteriori estimation with a priori distribution of blurs derived from the multichannel framework and a priori distribution of original images defined by the variational integral. This stochastic approach enables us to recover the blurs and the original image from channels severely corrupted by noise. We observe that the exact knowledge of the blur size is not necessary, and we prove that translation misregistration up to a certain extent can be automatically removed in the restoration process.
\end{abstract}

Index Terms-Image restoration, maximum a posteriori (MAP) estimator, multichannel blind deconvolution, subspace methods, variational integral.

\section{INTRODUCTION}

$\mathbf{I}$ $\mathrm{N}$ MANY applications, such as microscopy imaging, remote sensing, and astronomical imaging, observed images are often degraded by blurring. Examples of the most common sources of blur are atmospheric turbulence, relative motion between a camera, and an object or wrong focus. Restoration of the degraded images is a necessary step that precedes further image analysis.

First, a proper mathematical model that simulates the acquisition system is required. Images may be regarded as either deterministic or stochastic signals, blurred by linear or nonlinear processes and corrupted with additive or multiplicative noise. In the sequel, we assume a linear filter model with additive uncorrelated noise, i.e.

$$
z(x)=(h * u)(x)+n(x)
$$

where $z, h, u$, and $n$ are the degraded image, system PSF (blur), original image, and noise, respectively, and $*$ denotes convolution. This model accurately describes many common degradations and that justifies its frequent use.

The amount of a priori information about the degradation, like the size or shape of blurring functions and the noise parameters, significantly influences the success of restoration. When the blur function is known, many conventional approaches have been developed to compensate for the distortion [1].

Manuscript received January 27, 2004; revised July 23, 2004. This work was supported by the Grant Agency of the Czech Republic under Grant 102/04/0155. The associate editor coordinating the review of this manuscript and approving it for publication was Dr. Robert D. (G. E.) Nowak.

The authors are with the Institute of Information Theory and Automation, Academy of Sciences of the Czech Republic, 18208 Prague 8, Czech Republic (e-mail: sroubekf@utia.cas.cz; flusser@utia.cas.cz).

Digital Object Identifier 10.1109/TIP.2005.849322
The problem is ill posed, and, to overcome this difficulty, it is common to use regularization. When the blur is unknown, we talk about blind image restoration. A basic survey of different blind restoration techniques is given in [2]. Most of the methods are iterative or recursive. They involve regularization terms based on available prior information which assure various statistical properties of the image and constrains the estimated image and/or restoration filter. As in the nonblind case, regularization is required to improve stability. For images with sharp changes of intensity, the appropriate regularization is based on variational integrals. A special case of the variational integral, total variation, was first proposed in [3]. Minimization of the variational integrals preserves edges and fine details in the image and it was applied to image denoising [4]-[6] and to blind restoration [7]-[9], as well. Since the blind case is strongly ill posed, all the methods suffer from convergence and stability problems. If the images are smooth and homogeneous, an autoregressive model can be used to describe the measuring process. The autoregressive model simplifies the blind problem by reducing the number of unknowns and several techniques were proposed for finding its solution [10]-[12].

There are many applications, where different blurred versions of the same original image are observed through multiple acquisition channels. We distinguish in general two multichannel (MC) models: the single-input multiple-output (SIMO) model and the multiple-input multiple-output (MIMO) model. The SIMO model (see Fig. 1) is typical for one-sensor imaging under varying environment conditions, where individual channels represent the conditions at time of acquisition. The MIMO model refers, for example, to multisensor imaging, where the channels represent different spectral bands or resolution levels. Color images are the special case of the MIMO model. An advantage of MIMO is the ability to model cross-channel degradations which occur in the form of channel crosstalks, leakages in detectors, and spectral blurs. Many techniques for solving the MIMO problem were proposed and could be found in [13]-[16]. In the sequel, we confine ourselves to the SIMO model exclusively and any reference to the term MC denotes the SIMO model. Sometimes the SIMO model is referred to in the literature as a multiframe model. Following the above notation, we define the SIMO model as:

$$
z_{k}(x)=\left(h_{k} * u\right)(x)+n_{k}(x), \quad k=1, \ldots, K
$$

where $K$ is the number of channels. Examples of such MC measuring processes are common, e.g., in remote sensing and astronomy, where the same scene is observed at different time instants through a time-varying inhomogeneous medium such as the atmosphere; in confocal microscopy, where images of 
the same sample are acquired at different focusing lengths; or in broadband imaging through a physically stable medium, but which has a different transfer function at different frequencies. Nonblind MC restoration is potentially free of the problems arising from the zeros of blurs. The lack of information from one blur in one frequency can be supplemented by the information at the same frequency from the others. Intuitively, one may expect that the blind restoration problem is also simplified by the availability of different channels. Two classes of MC blind image restoration algorithms exist. Extensions of single-channel blind restoration approaches form the first class, but since they suffer from similar drawbacks as their single-channel counterparts, they are of not much interest. The other class consists of intrinsic MC approaches and will be considered here.

One of the earliest intrinsic multichannel blind deconvolution (MBD) methods [17] was designed particularly for images blurred by atmospheric turbulence. Harikumar et al. [18] proposed an indirect algorithm, which first estimates the blur functions and then recovers the original image by standard nonblind methods. The blur functions are equal to the minimum eigenvector of a special matrix constructed by the blurred images. Necessary assumptions for perfect recovery of the blur functions are noise-free environment and channel coprimeness, i.e., a scalar constant is the only common factor of the blurs. Giannakis et al. [19] (and at the same time Harikumar et al. [20]) developed another indirect algorithm based on Bezout's identity of coprime polynomials which finds restoration filters and by convolving the filters with the observed images recovers the original image. Both algorithms are vulnerable to noise and even for a moderate noise level restoration may break down. In the latter case, noise amplification can be attenuated to a certain extent by increasing the restoration filter order, which comes at the expense of deblurring. Pai et al. [21], [22] suggested two MC restoration algorithms that, contrary to the previous two indirect algorithms, estimate directly the original image from the null space or from the range of a special matrix. Another direct method based on the greatest common divisor was proposed by Pillai et al. in [23]. In noisy cases, the direct algorithms are more stable than the indirect ones. Interesting approaches based on the ARMA model are given in [24], [25]. MC blind deconvolution based on the Bussgang algorithm was proposed in [26], which performs well on spatially uncorrelated data, such as binary text images and spiky images. Most of the algorithms lack the necessary robustness since they do not include any noise assumptions (except ARMA and Bussgang) in their derivation and miss regularization terms. Recently, we have proposed an iterative MC algorithm [27] that performs well even on noisy images. It is based on least-squares deconvolution by anisotropic regularization of the image and between-channel regularization of the blurs.

Unfortunately, all the above mentioned multichannel blind deconvolution methods contain two ultimate but unrealistic assumptions. They require exact knowledge of the PSFs support size and individual channels are supposed to be perfectly spatially aligned (registered). These strong assumptions are seldom true in practice and in fact they have prevented the usage of multichannel blind deconvolution methods in real applications.

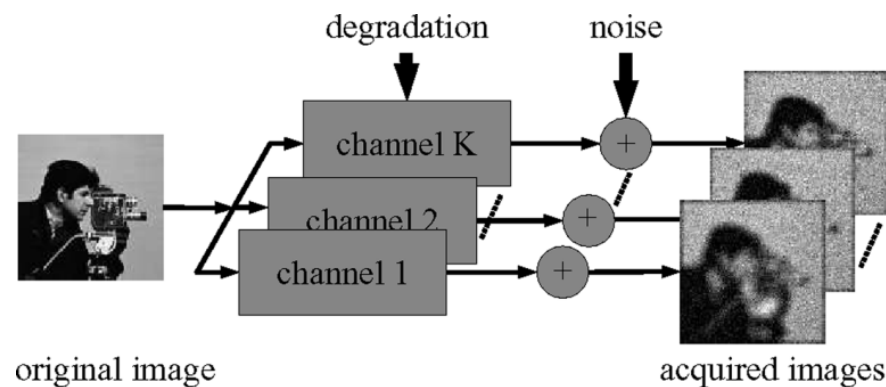

Fig. 1. Single-input multiple-output model: The original scene is captured by $K$ different channels which are subject to various degradations.

A realistic image acquisition model contains spatial coordinate transformations $\tau_{k}$ that describe geometric differences between the original scene and the $k$ th channel

$$
z_{k}\left(\tau_{k}(x)\right)=\left(h_{k} * u\right)(x)+n_{k}(x), \quad k=1, \ldots, K .
$$

Image deformations $\tau_{k}$ originate from the fact that the channels are two-dimensional (2-D) projections of the three-dimensional world, generally acquired from different viewpoints and/or with different camera orientation. In simple cases, $\tau_{k}$ is limited to rotation and translation, but, in real applications, complex nonlinear deformations may be present, too. Image restoration then consists of two stages: image registration, which brings the channels into spatial alignment, followed by multichannel blind deconvolution.

There have been published hundreds of image registration methods (see [28] for the most recent survey) and even special algorithms for registering blurred channels were developed [29]-[34]. Despite this effort, perfect registration accuracy can rarely be achieved, namely in the case of blurred and noisy images. The registration error results in a slight between-channel shift of up to a few pixels, which cannot be further compensated in the registration stage. Thus, channel-to-channel registration simplifies (3) to the form

$$
z_{k}\left(x+a_{k}\right)=\left(h_{k} * u\right)(x)+n_{k}(x), \quad k=1, \ldots, K
$$

where $a_{k}$ is a small unknown alignment error.

Model (4) also applies to numerous practical tasks directly without the preceding registration process. Such situations typically occur when the camera is subject to vibrations or in multitemporal imaging when the camera slightly moves between consecutive channel acquisitions and/or the scene is not perfectly still.

The deconvolution methods mentioned above cannot restore images degraded according to model (4). If they were applied, the channel misregistrations would lead to strong artifacts in the restored image.

In this paper, we introduce the first MBD method which does not require perfect alignment of the individual channels and the knowledge of the blur size. Exploiting the stochastic model and the Bayes rule in Section IV, we express the a posteriori probability of the original image in terms of the conditional probability and two a priori probabilities, which are derived from properties of bounded variation functions and from the MC framework. An alternating minimization (AM) algorithm 
as a solution to a maximum a posteriori probability (MAP) estimator is also given here. In Section V, we examine the minimization algorithm for its ability to alleviate the blur-oversized problem and demonstrate its convergence properties. We illustrate that the channel misalignment can be perfectly neutralized by properly oversizing the blur support in Section VI.

\section{NOTATION}

We use the following conventions throughout this paper:

$$
\begin{aligned}
& \mathbb{N}^{2} \\
& u: \mathbb{N}^{2} \rightarrow \mathbb{R} \\
& S_{u} \equiv\left(S_{u}^{1}, S_{u}^{2}\right) \\
& x=(i, j) \in \mathbb{N}^{2} \\
& u(x) \equiv u(i, j) \\
& \mathbf{u} \equiv
\end{aligned}
$$$$
\text { 2-D space of integers; }
$$$$
\text { image function with a finite rectangular }
$$$$
\text { support; }
$$$$
\text { support size of the image } u \text {; }
$$$$
\text { position at the } i \text { th row and the } j \text { th column }
$$
in the image;

image value at the position $x$; $\left[u(1,1), u(2,1), \ldots, u\left(S_{u}^{1}, 1\right), u(1,2)\right.$, $\left.\ldots, u\left(S_{u}^{1}, S_{u}^{2}\right)\right]^{T}$ image column vector, lowercase bold letters;

C

$\|\cdot\|$ matrix, uppercase bold letters; $l^{2}$ norm.

We endow the vector space $\mathbb{N}^{2}$ with the following operators: "+" and "-" $(i, j)+k$ $\prod(i, j)$ $(i, j)<(k, l)$ defined in a standard way; abbreviated form for $(i, j)+(k, k)$; $\equiv i \cdot j$; $\equiv\{i<k \wedge j<l\}$ and similarly other binary relations " $>$," "=," etc.

For our next discussion, it is necessary to define convolution with a variable output support in matrix-vector notation. We follow the definition in [18]. Let $h(x)$ and $u(y)$ be two images with support $1 \leq x \leq S_{h}=\left(S_{h}^{1}, S_{h}^{2}\right)$ and $1 \leq y \leq$ $S_{u}=\left(S_{u}^{1}, S_{u}^{2}\right)$, respectively, and $A=\left(a_{1}, a_{2}\right), B=\left(b_{1}, b_{2}\right)$ define an arbitrary output rectangle, where $A \leq B$. We separate $h$ column-wise and address individual columns as $h_{1}, \ldots, h_{S_{h}^{2}}$. We denote by $\mathbf{C}_{S}^{A, B}\{h\}$ a Toeplitz-block-Toeplitz matrix of size $\prod(B-A+1) \times \prod S_{u}$ such that the concatenated result of convolution $\left[\sum_{x} h(y-x) u(x)\right]_{(A \leq y \leq B)}$ is equal to $\mathbf{C}_{S_{u}}^{A, B}\{h\} \mathbf{u}$. This is given by

$$
\begin{aligned}
\mathbf{C}_{S_{u}}^{A, B}\{h\} & \underbrace{\left(\begin{array}{cccc}
\mathbf{D}_{S_{u}^{1}}^{a_{1}, b_{1}}\left\{h_{a_{2}}\right\} & \cdots & \mathbf{D}_{S_{u}^{1}}^{a_{1}, b_{1}} & \left\{h_{a_{2}-S_{u}^{2}+1}\right\} \\
\mathbf{D}_{S_{u}^{1}, b_{1}}^{a_{1}}\left\{h_{a_{2}+1}\right\} & \cdots & \mathbf{D}_{S_{u}^{1}}^{a_{1}, b_{1}} & \left\{h_{a_{2}-S_{u}+2}\right\} \\
\vdots & \vdots & \vdots \\
\mathbf{D}_{S_{u}^{1}}^{a_{1}, b_{1}}\left\{h_{b_{2}}\right\} & \cdots & \mathbf{D}_{S_{u}^{1}}^{a_{1}, b_{1}} & \left\{h_{b_{2}-S_{u}+1}\right\}
\end{array}\right)}_{=}
\end{aligned}
$$

and

$$
\begin{aligned}
\mathbf{D}_{s}^{a, b}\left\{h_{j}\right\} & \\
\quad= & \left(\begin{array}{cccc}
h(a, j) & h(a-1, j) & \cdots & h(a-s+1, j) \\
h(a+1, j) & h(a, j) & \cdots & h(a-s+2, j) \\
\vdots & \vdots & \vdots & \vdots \\
h(b, j) & h(b-1, j) & \cdots & h(b-s+1, j)
\end{array}\right)
\end{aligned}
$$

where $h(i, j)=0$ for $(i<1) \vee\left(i>S_{h}^{1}\right) \vee(j<1) \vee\left(j>S_{h}^{2}\right)$ and the size of $\mathbf{D}_{s}^{a, b}\left\{h_{j}\right\}$ is $(b-a+1) \times s$.

\section{PROBLEM Formulation}

We first define the SIMO degradation model in the discrete domain $\mathbb{N}^{2}$ as follows. Suppose that an original (input) image $u(x)$ has support $1 \leq x \leq S_{u}$. The input image propagates through $K$ different channels that behave as linear filters each with a finite impulse response (blurs) $h_{k}, k \in\{1, \ldots, K\}$. Let the maximum support of the blurs be $S_{h}$. In each channel, the image is further degraded with additive white Gaussian noise (AWGN) $n_{k}$ of zero mean and variance $\sigma_{k}^{2}$ and shifted by $t_{k} \in$ $\mathbb{N}^{2}, t_{k} \geq 0$. Let $S_{t}$ denote the maximum observed shift. On the output, we receive degraded and shifted images $z_{k}(x)$ with minimum support $1 \leq x \leq S_{z}$, where $S_{z}=S_{u}-S_{h}-S_{t}+1$. The whole model can be expressed as

$$
\begin{aligned}
& z_{k}(x)= \sum_{1 \leq y \leq S_{u}-S_{h}+1} \delta_{t_{k}}\left(x-y+S_{t}+1\right) \\
& \times \sum_{1 \leq z \leq S_{u}} h_{k}\left(y-z+S_{h}\right) u(z)+n_{k}(x)
\end{aligned}
$$

where $x, y, z \in \mathbb{N}^{2}$, and $\delta_{t_{k}}$ is the delta function at $S_{t}-t_{k}+1$. By concatenating columns of the images, we can rewrite the previous equation in matrix-vector notation as

$$
\mathbf{z}_{k}=\mathbf{T}_{k} \mathbf{H}_{k} \mathbf{u}+\mathbf{n}_{k}
$$

where $\mathbf{z}_{k}, \mathbf{u}$, and $\mathbf{n}_{k}$ are corresponding column image vectors. $\mathbf{T}_{k}=\mathbf{C}_{S_{u}-S_{h}+1}^{S_{t}+1, S_{u}-S_{h}+1}\left\{\delta_{t_{k}}\right\}$ is of size $\prod\left(S_{u}-S_{h}-S_{t}+1\right) \times$ $\prod\left(S_{u}-S_{h}+1\right)$ and $\mathbf{H}_{k}=\mathbf{C}_{S_{u}}^{S_{h}, S_{u}}\left\{h_{k}\right\}$ is of size $\prod\left(S_{u}-\right.$ $\left.S_{h}+1\right) \times \prod S_{u}$. Both matrices are constructed according to (5) and perform discrete convolution with the reduced output size. We refer to this type of convolution as "valid," since the result is defined only on the area where both convolution arguments are properly defined. It is easy to verify that the matrix product $\mathbf{T}_{k} \mathbf{H}_{k}=\mathbf{G}_{k}$ denotes "valid" convolution with a mask $g_{k}(x)=$ $h_{k}\left(x-t_{k}\right)$ of size $S_{g}=S_{h}+S_{t}$. This mask is a shifted version of the original blur $h_{k}$. By concatenating the output vectors $\mathbf{z} \equiv$ $\left[\mathbf{z}_{1}^{T}, \ldots \mathbf{z}_{K}^{T}\right]^{T}$ and the shifted blur vectors $\mathbf{g} \equiv\left[\mathbf{g}_{1}^{T}, \ldots, \mathbf{g}_{K}^{T}\right]^{T}$, the $\mathrm{MC}$ model can be rewritten in two equivalent forms

$$
\mathbf{z}=\mathbf{G u}+\mathbf{n}=\mathbf{U g}+\mathbf{n}
$$

where $\mathbf{G} \equiv\left[\mathbf{G}_{1}^{T}, \ldots, \mathbf{G}_{K}^{T}\right]^{T}, \mathbf{n} \equiv\left[\mathbf{n}_{1}^{T}, \ldots, \mathbf{n}_{K}^{T}\right]^{T}$, and $\mathbf{U}$ is a block-diagonal matrix with $K$ blocks each performing convolution with the image $u$, i.e.

$$
\mathbf{U} \equiv \underbrace{\left(\begin{array}{ccc}
\mathbf{C}_{S_{g}}^{S_{g}, S_{u}}\{u\} & \cdots & \mathbf{0} \\
\vdots & \ddots & \vdots \\
\mathbf{0} & \cdots & \mathbf{C}_{S_{g}}^{S_{g}, \dot{S}_{u}\{u\}}
\end{array}\right)}_{K \text { blocks }}
$$

We have obtained a standard MC convolution model and all conclusions for blur restoration in [18] and [19] apply also to our shifted version. When noise is omitted, it follows from (6) that the "valid" convolution matrix $\mathbf{Z}_{k} \equiv \mathbf{C}_{S_{\bar{g}}}^{S_{\bar{g}}, S_{z}}\left\{z_{k}\right\}$ for some arbitrary support $S_{\bar{g}}$ is given by

$$
\mathbf{Z}_{k}=\mathcal{U} \mathcal{G}_{k}
$$


where $\mathcal{U} \equiv \mathbf{C}_{S_{g}+S_{\bar{g}}-1}^{S_{g}+S_{\bar{g}}-1, S_{u}}\{u\}$ and $\mathcal{G}_{k} \equiv \mathbf{C}_{S_{\bar{g}}}^{1, S_{g}+S_{\bar{g}}-1}\left\{g_{k}\right\}$. The above equality determines the rank property of $\mathbf{Z}_{k}$ and is utilized in the following lemma.

First, we recall an important definition from [19]. Let $\tilde{h}_{k}\left(z_{1}, z_{2}\right)$ denote the 2-D $z$ transform of the blur $h_{k}$. The polynomials $\left\{\tilde{h}_{k}\left(z_{1}, z_{2}\right)\right\}$ are called "weakly coprime" if their only common factor is a scalar constant.

Lemma 1: Suppose that $K \geq 2,\left\{\tilde{h}_{k}\left(z_{1}, z_{2}\right)\right\}_{k=1}^{K}$ are weakly coprime, $\mathcal{U}$ in (7) has full column rank and the noise term is not present in (6). Then, all solutions $\left\{\overline{\mathbf{g}}_{k}\right\}$ to

$$
\mathbf{Z}_{i} \overline{\mathbf{g}}_{j}-\mathbf{Z}_{j} \overline{\mathbf{g}}_{i}=\mathbf{0}, \quad 1 \leq i<j \leq K
$$

have the form

$$
\overline{\mathbf{g}}_{k}= \begin{cases}\mathbf{C}_{S_{g}, S_{\bar{g}}}^{1}\{f\} \mathbf{g}_{k}, & \text { if } S_{\bar{g}} \geq S_{h}+S_{t} \\ \alpha \mathbf{g}_{k}, & \text { if } S_{\bar{g}}=S_{h}+S_{t} \\ \emptyset, & \text { otherwise }\end{cases}
$$

where $f$ is some spurious factor of size $S_{\bar{g}}-S_{h}-S_{t}+1$ and $\alpha$ is some scalar.

The proof is similar in nature to the proof given in [18] except that $S_{t}$ is included in size constraints as discussed below. The above lemma states that in the noiseless case, if the estimated blur size $S_{\bar{g}}$ is equal to the sum of the maximum size of the original blurs $S_{h}$ and the maximum shift $S_{t}$, then the true shifted blurs can be recovered precisely except to some scalar factor. This magnitude ambiguity can be resolved by stipulating, e.g., $\sum_{x} h_{k}(x)=1$, which is a standard energy preserving assumption. For oversized $S_{\bar{g}}$, the solutions lie in a subspace of dimension $\prod\left(S_{\bar{g}}-S_{g}+1\right)$. The first assumption that the blurs are weakly coprime is satisfied for many practical cases, since the necessary channel disparity is mostly guaranteed by the nature of the acquisition scheme and random processes therein. Refer to [18] for a relevant discussion. The second assumption of full column rank is also a mild one. For persistently exciting ${ }^{1} u$, the matrix $\mathcal{U}$ has full column rank provided that it has more rows than columns. Let us assume that the blur size is correctly estimated, i.e., $S_{\bar{g}}=S_{g}$, then $\mathcal{U}$ is of size $\prod\left(S_{u}-2\left(S_{g}+1\right)\right) \times \prod\left(2 S_{g}-1\right)$ from which follows a size constraint $\prod\left(S_{u}-2\left(S_{h}+S_{t}+1\right)\right) \geq$ $\prod\left(2\left(S_{h}+S_{t}\right)-1\right)$. Generally, $u$ is much larger than $h$ and the size constraint is violated only if the channel shift is for example $S_{t}>\left(S_{u}+3\right) / 4-S_{h}$. To rephrase and simplify the condition, $\mathcal{U}$ does not have full column rank if $S_{t}>S_{u} / 4$.

There are $K(K-1) / 2$ equations in (8), and after stacking them into one system, we get

$$
\mathcal{Z} \overline{\mathrm{g}}=\mathbf{0}
$$

where $\overline{\mathrm{g}} \equiv\left[\overline{\mathrm{g}}_{1}^{T}, \ldots, \overline{\mathrm{g}}_{K}^{T}\right]^{T}$

$$
\begin{aligned}
& \mathcal{Z} \equiv\left(\begin{array}{lll}
\mathcal{Z}_{1}^{T} & \ldots & \mathcal{Z}_{K-1}^{T}
\end{array}\right)^{T}
\end{aligned}
$$

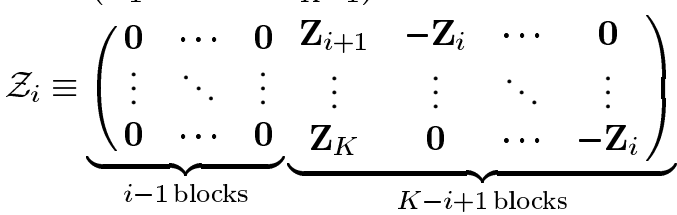

for $i=1, \ldots, K-1$.

\footnotetext{
${ }^{1}$ Function $u$ is called persistently exciting for size $S_{g}$ if $u * g$ is different from zero for any $g \neq 0$, which is almost certainly true for real images if $S_{g} \ll S_{u}$.
}

The motivation behind Lemma 1 is to reduce the problem of identifying the blurs to a null-space problem, where the dimension of the null-space of $\mathcal{Z}$ is $\prod\left(S_{\bar{g}}-S_{g}+1\right)$.

\section{MAP BLIND DECONVOLUTION}

Adopting a stochastic approach, the restoration problem can be formulated as a MAP estimation. We assume that the images $\mathbf{u}, \mathbf{g}$, and $\mathbf{z}$ are random vector fields with given probability density functions (pdf) $p(\mathbf{u}), p(\mathbf{g})$, and $p(\mathbf{z})$, respectively, and we look for such realizations of $\mathbf{u}$ and $\mathbf{g}$, which maximize the $a$ posteriori probability $p(\mathbf{u}, \mathbf{g} \mid \mathbf{z})$. We assume that $\mathbf{u}$ and $\mathbf{h}$ are uncorrelated then, according to the Bayes rule, the relation between a priori densities $p(\mathbf{u}), p(\mathbf{g})$ and the a posteriori density is $p(\mathbf{u}, \mathbf{g} \mid \mathbf{z})=p(\mathbf{z} \mid \mathbf{u}, \mathbf{g}) p(\mathbf{u}) p(\mathbf{g}) / p(\mathbf{z})$. The pdf $p(\mathbf{z})$ is a constant and can, thus, be omitted. The conditional pdf $p(\mathbf{z} \mid \mathbf{u}, \mathbf{g})$ follows from our model (6) and from our assumption of AWGN, i.e.

$$
p(\mathbf{z} \mid \mathbf{u}, \mathbf{g}) \propto \exp \left\{-\frac{1}{2}(\mathbf{z}-\mathbf{G u})^{T} \Sigma^{-1}(\mathbf{z}-\mathbf{G u})\right\}
$$

where $\Sigma$ is the noise diagonal covariance matrix with $\left\{\sigma_{k}^{2}\right\}_{k=1}^{K}$ on the corresponding positions on the main diagonal. If the same noise variance $\sigma^{2}$ is assumed in each channel, $\Sigma^{-1}$ reduces to a scalar $\sigma^{-2}$.

\section{A. A Priori Distribution of the Original Image}

The necessity of meaningful a priori probabilities becomes often Achilles' heel of Bayesian approaches. Several different forms of the image a priori probabilities were proposed in the literature. Some are suitable only for a specific class of images and others are more general. The classical form chooses the Laplacian operator as the inverse of the covariance matrix of $u$, i.e., $p(\mathbf{u}) \propto \exp \left(-\mathbf{u}^{T} \mathbf{L} \mathbf{u}\right)$, where $\mathbf{L}$ denotes the discrete Laplacian operator. The exponent $\mathbf{u}^{T} \mathbf{L u}$ is the discretization of $\int|\nabla u(x)|^{2} d x$, where $\nabla u$ denotes the gradient of $u$. Apart from easy implementation, this pdf is not suitable for the prior model, since the $L_{2}$ norm of the image gradient penalizes too much the gradients corresponding to edges and an oversmoothing effect is observed. In real images, object edges create sharp steps that appear as discontinuities in the intensity function. It is the space of bounded variation (BV) functions that is widely accepted as a proper setting for real images. Rudin et al. [3] first demonstrated very good anisotropic denoising properties of the total variation $\int|\nabla u(x)| d x$. Existence and uniqueness of the minimum of total variation is possible only in the BV space, in which case $\nabla u$ denotes the gradient of $u$ in the distributional sense. The same holds true for a more general case of convex functions of measures $\int \phi(|\nabla u(x)|) d x$, where $\phi$ is a strictly convex, nondecreasing function that grows at most linearly. Examples of $\phi(s)$ are $s$ (total variation), $\sqrt{1+s^{2}}-1$ (hypersurface minimal function), or $\log (\cosh (s))$. For nonconvex functions, nothing can be said about the existence of the minimum. Nevertheless, nonconvex functions, such as $\log \left(1+s^{2}\right), s^{2} /\left(1+s^{2}\right)$ or $\arctan \left(s^{2}\right)$ (Mumford-Shah functional [35]), are often used since they provide better results for segmentation problems.

Our a priori image distribution consists of the convex function of measures. The function is highly nonlinear and to overcome this difficulty we follow a half-quadratic scheme proposed 
in [36] and [37], which introduces an auxiliary variable. Special attention must be paid to the discretization of the image gradient $\nabla u(x)$ and relaxation of $\phi$. If a second-order centered approximation of the first derivative is used, the prior pdf takes the form

$$
\begin{aligned}
p(\mathbf{u}, v) \propto & \exp \left\{\frac{1}{2} \sum_{i, j} v\left(i+\frac{1}{2}, j\right)(u(i+1, j)-u(i, j))^{2}\right. \\
& \left.+v\left(i, j+\frac{1}{2}\right)(u(i, j+1)-u(i, j))^{2}\right\} \\
\equiv & \exp \left\{-\frac{1}{2} \mathbf{u}^{T} \mathbf{L}(v) \mathbf{u}\right\}
\end{aligned}
$$

where $v$ is given by

$$
\begin{aligned}
v(i+\zeta, j+\zeta) & =\frac{\phi^{\prime}(|u(i+2 \zeta, j+2 \zeta)-u(i, j)|)}{|u(i+2 \zeta, j+2 \zeta)-u(i, j)|} \\
\zeta & =\left\{0, \frac{1}{2}\right\} .
\end{aligned}
$$

The auxiliary flux variable $v$ is similar to Geman's line process [38]. It denotes the edge strength between point $(i, j)$ and its neighbors $(i \pm 1, j),(i, j \pm 1)$. For example, in the case of the hypersurface minimal function $\phi(s)=\sqrt{1+s^{2}}-1$, the flux variable becomes $v(s)=\phi^{\prime}(s) / s=1 / \sqrt{1+s^{2}}$. Matrix $\mathbf{L}(v)$ is a positive semidefinite block tridiagonal matrix constructed by $v$ that performs shift-variant convolution with $v$. In the above discretization, the norm of the image gradient is variant to rotation. A more precise discretization is possible if we take into account the diagonal values.

\section{B. A Priori Distribution of the Blurs}

We derive the a priori distribution $p(\mathrm{~g})$ directly from the MC model. If the AWGN noise term $\mathbf{n}$ is present in model (6), then the left-hand side of (9) is not zero, but equal to a realization of a Gaussian process with zero mean and covariance $\mathcal{C}=\mathcal{G} \Sigma \mathcal{G}^{T}$. Matrix $\mathcal{G}$ takes the form of $\mathcal{Z}$ in (10) with $\mathbf{Z}_{i}$ replaced by $\mathbf{C}_{S_{z}}^{S_{g}, S_{z}}\left\{g_{i}\right\}$. Our first estimate of the a priori pdf $p^{\prime}(\mathrm{g})$ is then given by

$$
p^{\prime}(\mathbf{g}) \propto \exp \left\{-\frac{1}{2} \mathbf{g}^{T} \mathcal{Z}^{T} \mathcal{C}^{-1} \mathcal{Z} \mathbf{g}\right\} .
$$

From Lemma 1 , it follows that $\mathcal{Z}^{T} \mathcal{C}^{-1} \mathcal{Z}$ is close to singular and the number of eigenvalues that cluster around the noise variance is proportional to the degree of overestimation $\left(\prod\left(S_{\bar{g}}-S_{g}+1\right)\right)$. The expected blurs lie inside a subspace defined by eigenvectors that correspond to these eigenvalues. We propose to construct a priori $p(\mathbf{g})$ by constraining $p^{\prime}(\mathbf{g})$ to a set of admissible solutions. The set of admissible solutions is defined by our assumption that the blurs are positive and preserve energy; $A \equiv\left\{\mathrm{g} \mid g_{k}(x) \geq 0 \wedge \sum_{x} g_{k}(x)=1, k=1, \ldots, K\right\}$. We write

$$
p(\mathbf{g})= \begin{cases}p^{\prime}(\mathbf{g}), & \text { if } \mathbf{g} \in A \\ 0, & \text { otherwise. }\end{cases}
$$

This leads to a prior pdf that is data dependent. From a strictly theoretical point of view, one should use here a different set of input data but degraded by the same blurs as the data $\mathbf{z}$ in question, or use one part of the input data for $p(\mathbf{g})$ and perform restoration on the other part.

The main difficulties are connected with the matrix $\mathcal{C}$. The inverse of the matrix is not trivial and the matrix is constructed by the blurs $g$ that are to be estimated. One way is to use an iterative algorithm and update $\mathcal{C}$ by $\mathrm{g}$ estimated in the previous iteration. This iterative maximization of $p^{\prime}(\mathrm{g})$ w.r.t. $\mathrm{g}$ closely resembles the maximum-likelihood algorithm proposed by Harikumar [18]. However, this updating procedure is difficult to justify. We, therefore, propose to simplify $\mathcal{C}$ and approximated it by a diagonal matrix $\mathcal{D}$ such that $\operatorname{diag}(\mathcal{D})=\operatorname{diag}(\mathcal{C})$, where $\operatorname{diag}(\cdot)$ denotes the main diagonal of the matrix. The elements of $\mathcal{D}$ take the form $\sigma_{i}^{2}\left\|\mathrm{~g}_{j}\right\|^{2}+\sigma_{j}^{2}\left\|\mathrm{~g}_{i}\right\|^{2}$ for $1 \leq i<j \leq K$. The value of $\left\|\mathrm{g}_{i}\right\|^{2}$ is not known in advance, but a good initial approximation can be given. If $\mathrm{g} \in A$, then $\left(1 / \prod S_{g}\right) \leq\left\|\mathrm{g}_{i}\right\|^{2} \leq 1$, and we use the bottom limit for $\left\|\mathbf{g}_{i}\right\|^{2}$.

\section{AM-MAP Algorithm}

The a posteriori pdf $p(\mathbf{u}, \mathbf{g} \mid \mathbf{z})$ is composed of (11), (12), and (14) and turns out to be

$$
\begin{aligned}
p(\mathbf{u}, \mathbf{g} \mid \mathbf{z}) \propto \exp \{- & \frac{1}{2}\left((\mathbf{z}-\mathbf{G u})^{T} \Sigma^{-1}(\mathbf{z}-\mathbf{G u})\right. \\
& \left.\left.+\mathbf{u}^{T} \mathbf{L}(v) \mathbf{u}+\mathbf{g}^{T} \mathcal{Z}^{T} \mathcal{D}^{-1} \mathcal{Z} \mathbf{g}\right)\right\}
\end{aligned}
$$

for $\mathrm{g} \in A$ and zero otherwise. The MAP estimation is then equivalent to minimizing $E(\mathbf{u}, \mathbf{g})=-\log (p(\mathbf{u}, \mathbf{g} \mid \mathbf{z}))$ subjected to $\mathrm{g} \in A$. To find a minimizer of the energy function $E$, we perform alternating minimizations of $E$ over $\mathbf{u}$ and $\mathbf{g}$. The advantage of this scheme lies in its simplicity. Each term in (15) is convex (but not necessarily strictly convex, especially, when $\mathbf{g}$ is oversized) and the derivatives w.r.t. $\mathbf{u}$ and $\mathbf{g}$ can be easily calculated.

In summary, the AM-MAP algorithm alternates between two steps

1)

$$
\begin{aligned}
\mathbf{u}^{m+1} & =\arg \min _{\mathbf{u}} E\left(\mathbf{u}, \mathbf{g}^{m}\right) \\
& \Leftrightarrow\left(\mathbf{G}^{T} \Sigma^{-1} \mathbf{G}+\mathbf{L}(v)\right) \mathbf{u}=\mathbf{G}^{T} \Sigma^{-1} \mathbf{z}
\end{aligned}
$$

2)

$$
\begin{aligned}
\mathbf{g}^{m+1} & =\arg \min _{\mathbf{g} \in A} E\left(\mathbf{u}^{m+1}, \mathbf{g}\right) \\
& \Leftrightarrow\left(\mathbf{U}^{T} \Sigma^{-1} \mathbf{U}+\mathcal{Z}^{T} \mathcal{D}^{-1} \mathcal{Z}\right) \mathbf{g} \\
& =\mathbf{U}^{T} \Sigma^{-1} \mathbf{z} \wedge \mathbf{g} \in A .
\end{aligned}
$$

In step 1, the flux variable $v$ is updated according to (13).

Our AM approach is a variation on the steepest-descent algorithm. The search space is a concatenation of the blur subspace and the image subspace. The algorithm first descends in the image subspace and after reaching the minimum, i.e., $\nabla_{\mathbf{u}} E=0$, it advances in the blur subspace in the direction $\nabla_{\mathrm{g}} E$ orthogonal to the previous one, and this scheme repeats. We use the preconditioned conjugate gradient method (function $p c g$ in Matlab) to solve the unconstrained minimization problem (16) and fmincon (Optimization Toolbox) function 
to solve the constrained minimization problem (17). $E$ as a function of both variables $\mathbf{u}$ and $\mathbf{g}$ is not convex. We cannot, thus, guarantee that the global minimum is reached by the AM-MAP algorithm. Nevertheless, our thorough testing have shown good convergence properties of the algorithm for many real problems. In the following experiments, the hypersurface minimum function was used as $\phi(s)$, and the more precise discretization involving diagonal terms was implemented. We also assumed that the noise variance $\sigma_{k}^{2}$ is known and is the same in each channel. If this is not the case, the noise variance can be assessed by standard noise estimation methods or an approach of "trial and error" can be considered. The impact of wrong $\sigma_{k}^{2}$ can be easily observed. If the parameter is too small, i.e., we assume less noise, the restoration process begins to amplify noise in the image. If the parameter is too big, the restoration process starts to segment the image.

\section{Initial Guess}

Setting appropriately initial blurs can help our iterative algorithm to converge to the global minimum. This issue is important especially for the overestimated blur size. One can readily see that translated versions of the correct blurs give the same maximum probability $p(\mathbf{u}, \mathbf{g} \mid \mathbf{z})$ as long as they fit into our estimated blur size. We already know that the prior pdf of the blurs is unable to distinguish between the correct blurs and the correct blurs convolved with an arbitrary spurious factor. This makes a negative impact on the convergence mainly if the channel misalignment occurs, since new local minima appear for blurs that cope with the misalignment by convolving the correct blurs with an interpolating kernel. For example, to compensate a one-pixel shift between two channels, the suboptimal solution is to shift both blurs in the opposite direction by half a pixel and perform an interpolation, while the correct solution (global minimum) is to shift one of the estimated blurs by one pixel and leave the other. To get closer to the correct solution, we, thus, propose to set the initial blurs $\mathrm{g}^{0}$ to delta functions positioned at the centers of gravity of blurs $\tilde{\mathrm{g}}=\arg \max _{\mathrm{g}} p(\mathrm{~g})$. More precisely, if the images were blurred with energy preserving PSFs $\mathrm{g} \in A$, the centers of gravity $(\operatorname{cog})$ satisfy $\operatorname{cog}\left(g_{i}\right)-\operatorname{cog}\left(g_{j}\right)=$ $\operatorname{cog}\left(g_{i} * f\right)-\operatorname{cog}\left(g_{j} * f\right)$ for any $f$ that preserves energy. From Lemma 1 , it follows that our estimate $\tilde{g}_{i}$ is a good approximation of $g_{i} * f$, and we can, thus, calculate the relative positions of the centers of gravity. This technique enables us to compensate for the channel shifts right from the start of the algorithm and get away from the incorrect interpolated solutions.

\section{OVERSIZED BLURS}

It is difficult to analyze global convergence properties of the algorithm (16), (17) due to the nonlinear term $\mathbf{L}(v)$. Chan et al. in [9] transformed a similar alternating minimization problem into the Fourier domain and performed the analysis there. In the Fourier domain, it is difficult to apply the support constraint on the blurs, but in our case, the blur size plays a fundamental role in Lemma 1. The analysis should, thus, be performed in the $z$ transform domain but this is difficult to carry out.

We have run a series of experiments on simulated data with an incorrectly estimated blur size. A standard $128 \times 128$ "Lena" image was degraded with three random blurs of size $3 \times 3$ and with additive Gaussian noise of SNR $=20,30,40,50 \mathrm{~dB}$ $\left(\mathrm{SNR}=10 \log \left(\operatorname{var}(u) / \sigma^{2}\right)\right.$, where $\operatorname{var}(u)$ is the variance of the original image). The original image was recovered from each image triplet using the alternating minimization algorithm with the blur size set to $3 \times 3,4 \times 4,6 \times 6$, and $8 \times 8$, respectively. The percentage mean squared error of the estimated image $\overline{\mathbf{u}}$ defined as $\operatorname{PMSE}(\overline{\mathbf{u}})=100\|\overline{\mathbf{u}}-\mathbf{u}\| /\|\mathbf{u}\|$ was used as the evaluation measure at each iteration. Calculated PMSEs are summarized in Fig. 2. For less noisy data $(\mathrm{SNR}=40,50 \mathrm{~dB})$, the convergence rate is fast and not affected by the incorrect blur size estimation. In the case of SNR $=20,30 \mathrm{~dB}$, we observe a negative influence of the overestimated blur size on the convergence. However, not many practical applications provide data with such low SNRs.

\section{SHIFT-INVARIANT RESTORATION}

In this section, we illustrate the ability of the method to handle channels which are not registered. This is the most important advantage of the new technique.

The first experiment demonstrates the capability of the AM-MAP algorithm to recover the original image from two degraded and shifted versions thereof, when the maximum shift between the two channels is known. The standard $128 \times 128$ "Lena" image was degraded with two $5 \times 5$ blurs. One blurred image was shifted by $10 \times 20$ pixels and then both images were cropped to the same size; see Fig. 3. The AM algorithm was initialized with the correctly estimated blur size $15 \times 25$. The restored image and blurs are shown in Fig. 4. The blurs are perfectly recovered and properly shifted. The restored image matches the original on the area where data from both channels were available. The same experiment was conducted again but Gaussian noise SNR $=30 \mathrm{~dB}$ was added to the blurred and shifted input images in Fig. 3. Obtained results are depicted in Fig. 5 and illustrate satisfying restoration.

In the second experiment, we overestimated the blur size and proceeded as follows. The $250 \times 250$ test image in Fig. 6(a) was degraded with two different $5 \times 5$ blurs and noise of SNR $=50 \mathrm{~dB}$. One blurred image was shifted by $5 \times 5$ pixels and then both images were cropped to the same size; see Fig. 6(c). Contrary to the previous experiment, the shift was considered unknown and the AM-MAP algorithm was initialized with the overestimated blur size $12 \times 12$. The fused image and the estimated blur masks are shown in Fig. 7. Recovered blurs contain negligible spurious factors and are properly shifted to compensate for the misregistration. The fused image is by visual comparison much sharper than the input channels and very similar to the original, which demonstrates excellent performance.

We have also compared the performance of the AM-MAP algorithm with the performance of Pai's method [22] for different noise levels. The Pai approach directly recovers the original image by calculating the maximum singular vector of a special matrix. The $Q R$ decomposition is necessary for the construction of this matrix and the power method (or any other iterative method for eigenvector computation) is used to find the maximum singular vector, i.e., the original image. 


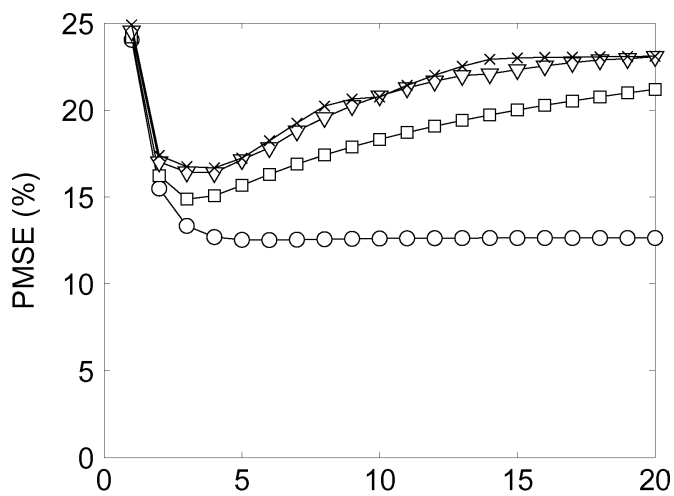

(a)

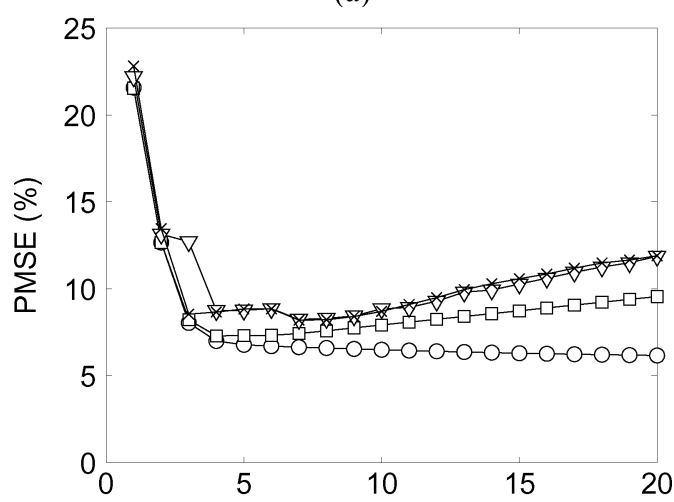

(b)

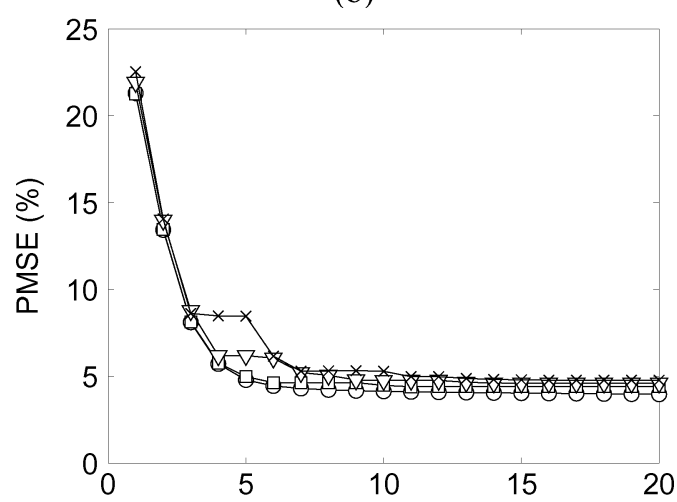

(c)

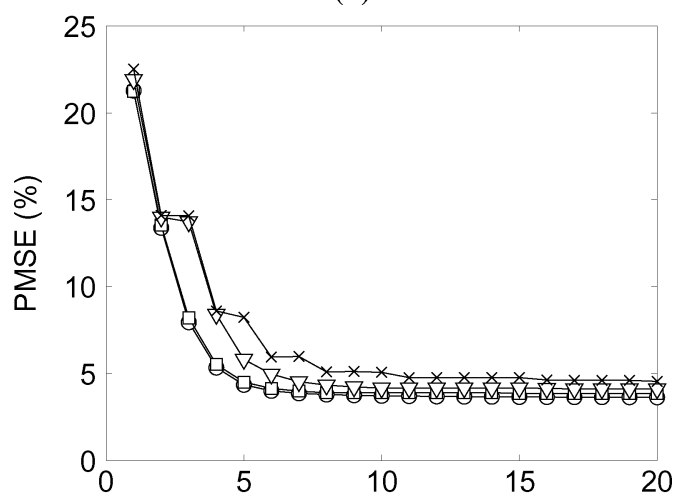

(d)

Fig. 2. PMSE of the estimated image as a function of iteration. The "Lena" image was degraded with three random blurs of size $3 \times 3$ and with AWGN of SNR (a) 20, (b) 30 , (c) 40, and (d) $50 \mathrm{~dB}$. The AM algorithm was executed with the estimated blur size $(\bigcirc) 3 \times 3$ (correct size), $(\square) 4 \times 4,(\nabla) 6 \times 6$, and $(\times)$ $8 \times 8$.
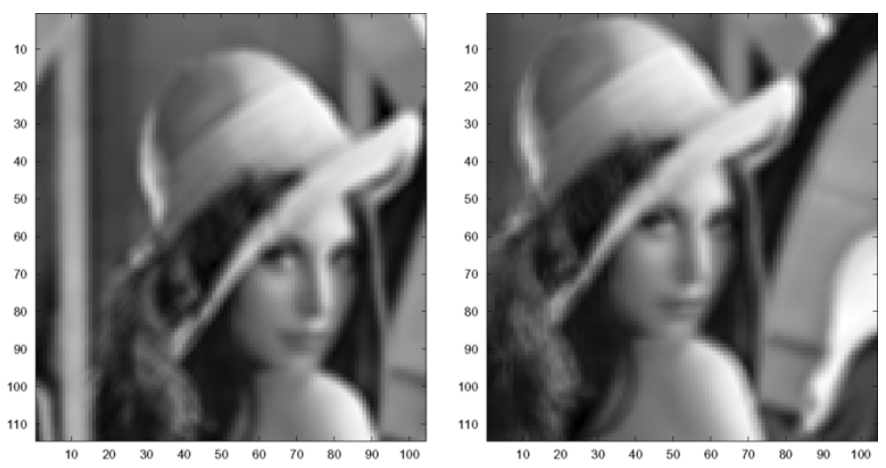

(a)
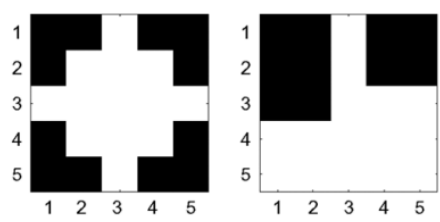

(b)

Fig. 3. (a) Input channels. "Lena" images degraded with two $5 \times 5$ blurs. Mutual translation between the images is 10 pixels vertically and 20 pixels horizontally. (b) Blurring masks.

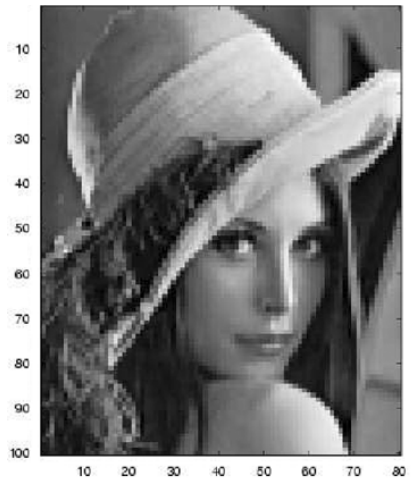

(a)
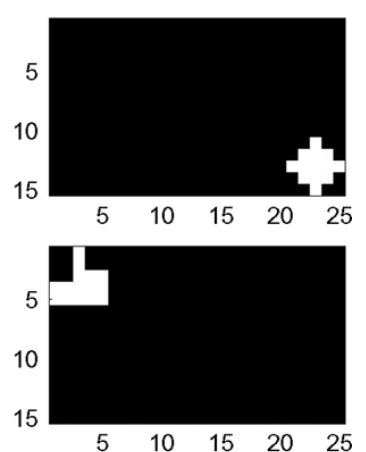

(b)
Fig. 4. Perfect noise-free AM restoration. (a) Recovered "Lena" image. (b) Recovered blurs and $10 \times 20$ shift between channels.

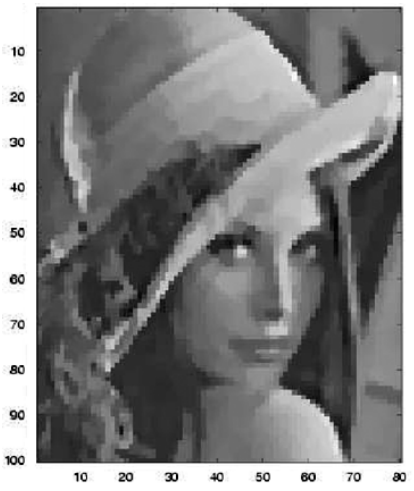

(a)
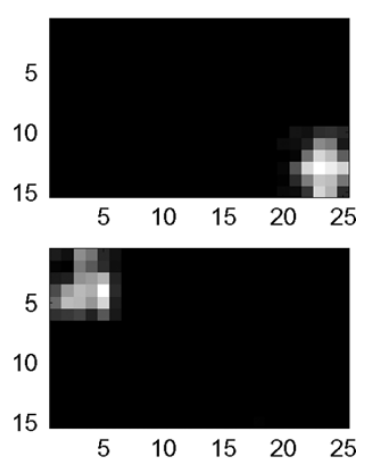

(b)
Fig. 5. Noisy AM restoration $(30 \mathrm{~dB})$. (a) Recovered "Lena" image. (b) Recovered blurs and $10 \times 20$ shift between channels.

Although the Pai method is not iterative in its definition, it requires numerical iterative methods and, thus, approaches the complexity of our inherently iterative algorithm. We used four randomly generated $3 \times 3$ blurs to obtain four blurred "Lena" 


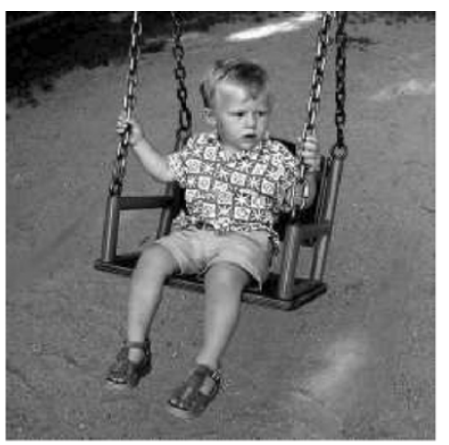

(a)

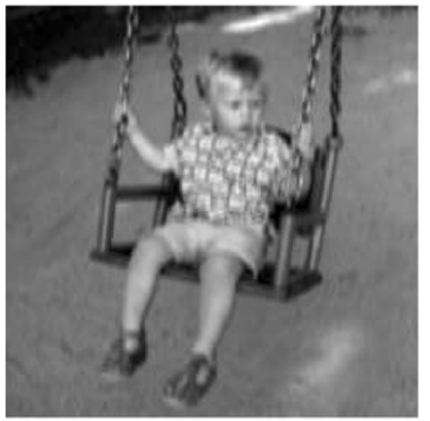

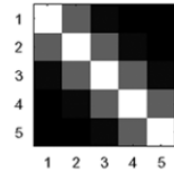

(b)

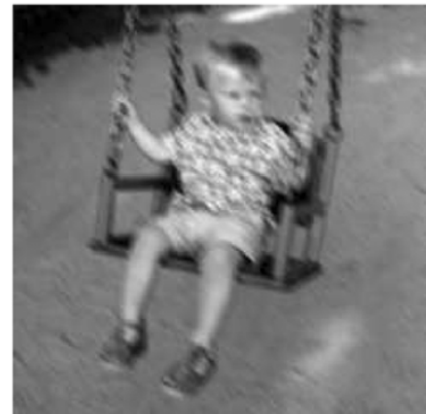

(c)

Fig. 6. (a) Original test image $250 \times 250$ pixels. (b) Two $5 \times 5$ PSFs. (c) Blurred and shifted images.

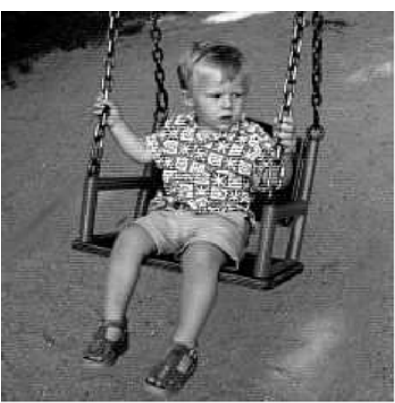

(a)
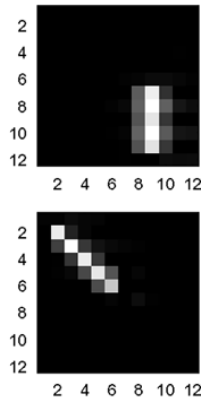

(b)
Fig. 7. Image restoration for the overestimated blur size. (a) Recovered image. (b) Estimated blur masks with the between-channel shift.

images. The images were then mutually translated so that centers of the images were in corners of a $5 \times 5$ square. Noise was added with $\mathrm{SNR}=10,20,30,40$, and $50 \mathrm{~dB}$, respectively. The maximum shift and the size of blurs were assumed to be known and, therefore, both methods were initialized with the correct blur size $8 \times 8$. For each SNR, the experiment was repeated with different blurs 10 times and stopped after 50 iterations in the AM-MAP case. The mean PMSE and standard deviation was calculated over these ten estimated images and plotted in Fig. 8. Clearly, the AM-MAP performs better then the Pai method for every SNR.

To evaluate the performance of the AM-MAP algorithm with respect to the knowledge of the channel misalignment, a different experiment was conducted. Degraded images were prepared in similar fashion as in the previous experiment but this time the translation between the channels varied from 0 to $5 \times 5$

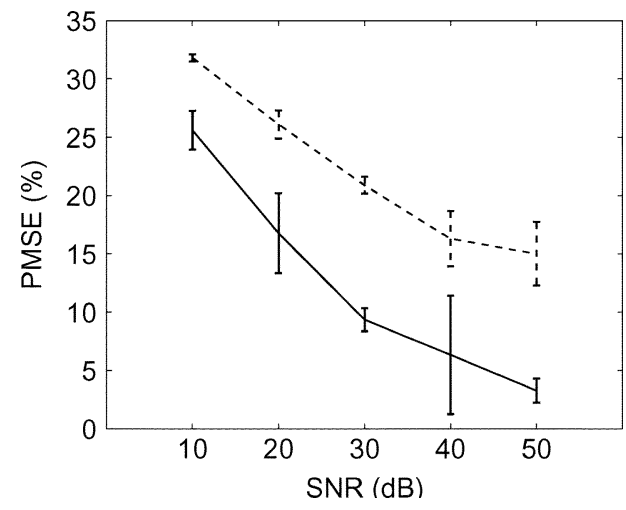

Fig. 8. Comparison of the (solid) AM-MAP algorithm and the (dashed) Pai method. Mean PMSE and (vertical abscissae) standard deviation of the restored images over ten different degradations and for different SNR.

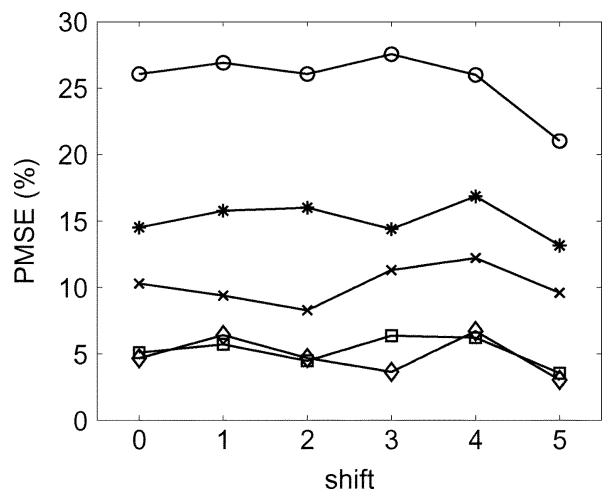

Fig. 9. AM-MAP algorithm performance on misaligned channels. Mean PMSE of restored images over ten different degradations for the channel misalignment 0 to 5 pixels and SNR (o) 10, (*) 20, ( $\times$ ) 30, ( $\square$ ) 40, and

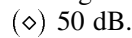

pixels to simulate inaccurate registration. For each shift, the experiment was repeated ten times with different blurs and was every time initialized to $8 \times 8$ blur size. The calculated mean PMSE is plotted in Fig. 9. PMSEs are almost constant, which demonstrates very good stability of the algorithm against the mask overestimation.

Finally, to demonstrate the power of the AM-MAP algorithm, we performed an experiment with real data. This experiment was motivated by many practical situations where we have to handle images degraded by random motion and/or vibration blur. This problem appears frequently in industrial visual inspection when the camera is mounted on a vibrating machine or when a stationary camera monitors vibrating environment. A text label (a part of a standard newspaper page) was attached to a vibrating machine. The label was monitored under poor light conditions by a standard digital camera mounted on a tripod. The camera exposure time was set at $1 / 15$ s which was comparable to the period of irregular vibrations of the machine. Three cropped images of the label acquired with the camera were used as the input channels of AM-MAP; see Fig. 10. The images were not binarized but only trasformed to grey-level images. Note strong motion blurs due to the machine movement and clear spatial misalignment of the channels. Since the shift and the size of the blurs were completely unknown and it was also difficult to estimate the upper bound, the AM-MAP algorithm was restarted with different parameters and the best results were 

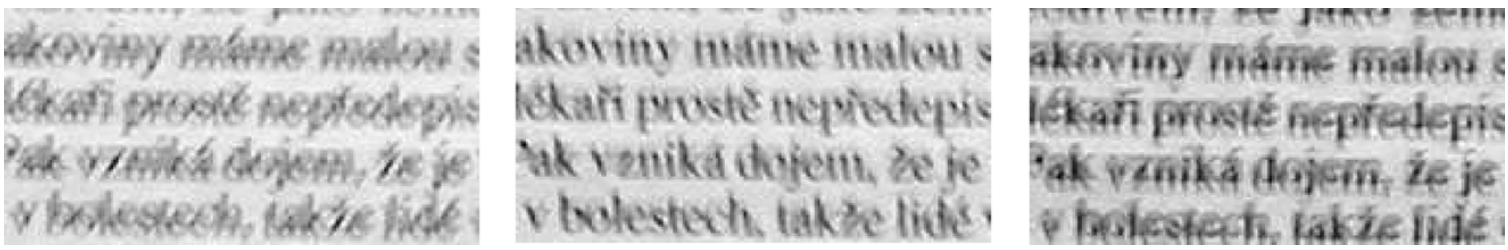

Fig. 10. Real data experiment. Three consecutive acquisitions of a text label attached to a vibrating machine. The grey-level images are cropped to $100 \times 200$ size. Shift blurs and spatial misalignment of the images are clearly visible.

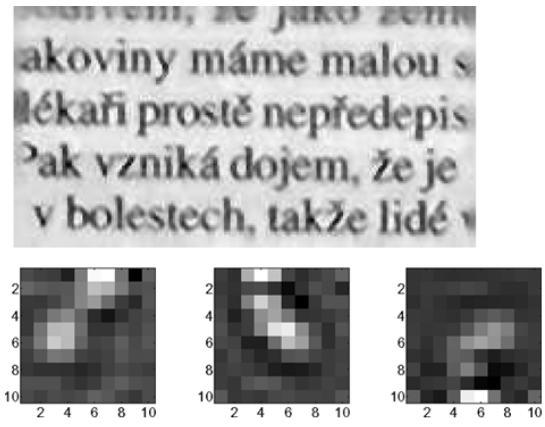

Fig. 11. Real data experiment. Reconstructed part of the label and the corresponding blurs (magnified) using the AM-MAP algorithm. The irregular vibration of the machine is well preserved in the blurs.

found for the blur size $10 \times 10$ and $\sigma^{2}=0.01$. We used the total variation in the a priori pdf to favor piecewise constant functions which is the case of the text label. The reconstructed label and the corresponding blur masks after 20 iterations are shown in Fig. 11. One can see that the restoration was successful (the text is clearly legible) and that the spatial misalignment inherent to this type of problems poses no threat to proper functionality of the algorithm. Observe that the restoration is slightly less successful near the image borders, especially close to the top edge, where only data from the third channel were available. Let us recall that no assumption about the shape of the blurring functions and no preprocessing of the input images were employed.

\section{CONCLUSION}

We have developed the iterative algorithm for multichannels blind deconvolution that searches for the MAP estimator. The prior density functions were derived from the variational integral defined on bounded variation functions and from the mutual relation of weakly coprime channels. The restoration is regularized with an anisotropic term for edge preservation and performs well on heavily degraded images with high SNR and shows better performance then the most recent multichannel method. We have also shown that the inaccurate registration of channels can be alleviated by properly overestimating the size of blurs. All previously published MBD methods assumed perfectly registered channels or required, though not specifically considered in their formulation, an exact knowledge of the channel misalignment. To our knowledge, this is the only method dealing explicitly with misregistration of images in the multichannel framework and providing a successful solution to this problem.

\section{REFERENCES}

[1] M. Banham and A. Katsaggelos, "Digital image restoration," IEEE Signal Process. Mag., vol. 14, no. 2, pp. 24-41, Mar. 1997.

[2] D. Kundur and D. Hatzinakos, "Blind image deconvolution," IEEE Signal Process. Mag., vol. 13, no. 3, pp. 43-64, May 1996.

[3] L. Rudin, S. Osher, and E. Fatemi, "Nonlinear total variation based noise removal algorithms," Phys. D, vol. 60, pp. 259-268, 1992.

[4] C. Vogel and M. Oman, "Fast, robust total variation-based reconstruction of noisy, blurred images," IEEE Trans. Image Process., vol. 7, no. 6, pp. 813-824, Jun. 1998.

[5] T. Chan, G. Golub, and P. Mulet, "A nonlinear primal-dual method for total variation-based image restoration," in SIAM J. Sci. Comput., vol. 20, Jul. 1999, pp. 1964-1977.

[6] A. B. Hamza, H. Krim, and G. Unal, "Unifying probabilistic and variational estimation," IEEE Signal Process. Mag., vol. 19, no. 5, pp. 37-47, Sep. 2002.

[7] T. Chan and C. Wong, "Total variation blind deconvolution," IEEE Trans. Image Process., vol. 7, no. 3, pp. 370-375, Mar. 1998.

[8] Y.-L. You and M. Kaveh, "Blind image restoration by anisotropic regularization," IEEE Trans. Image Process., vol. 8, no. 3, pp. 396-407, Mar. 1999.

[9] T. Chan and C. Wong, "Convergence of the alternating minimization algorithm for blind deconvolution," Linear Algebra Appl., vol. 316, no. 1-3, pp. 259-285, Sep. 2000.

[10] R. Lagendijk, J. Biemond, and D. Boekee, "Identification and restoration of noisy blurred images using the expectation-maximization algorithm," IEEE Trans. Acoust. Speech Signal Process., vol. 38, no. 7, pp. 1180-1191, Jul. 1990.

[11] S. Reeves and R. Mersereau, "Blur identification by the method of generalized cross-validation," IEEE Trans. Image Process., vol. 1, no. 3, pp. 301-311, Mar. 1992.

[12] M. Haindl, "Recursive model-based image restoration," in Proc. 15th Int. Conf. Pattern Recognition, vol. III, 2000, pp. 346-349.

[13] W. Zhu, N. Galatsanos, and A. Katsaggelos, "Regularized multichannel restoration using cross-validation," Graph. Models Image Process., vol. 57, no. 1, pp. 38-54, Jan. 1995.

[14] B. Tom, K. Lay, and A. Katsaggelos, "Multichannel image identification and restoration using the expectation-maximization algorithm," Opt. Eng., vol. 35, no. 1, pp. 241-254, Jan. 1996.

[15] A. Katsaggelos, K. Lay, and N. Galatsanos, "A general framework for frequency domain multi-channel signal processing," IEEE Trans. Image Process., vol. 2, no. 7, pp. 417-420, Jul. 1993.

[16] M. Kang, "Generalized multichannel image deconvolution approach and its applications," Opt. Eng., vol. 37, no. 11, pp. 2953-2964, Nov. 1998.

[17] T. Schulz, "Multiframe blind deconvolution of astronomical images," $J$. Opt. Soc. Amer. A, vol. 10, no. 5, pp. 1064-1073, May 1993.

[18] G. Harikumar and Y. Bresler, "Perfect blind restoration of images blurred by multiple filters: Theory and efficient algorithms," IEEE Trans. Image Process., vol. 8, no. 2, pp. 202-219, Feb. 1999.

[19] G. Giannakis and R. Heath, "Blind identification of multichannel FIR blurs and perfect image restoration," IEEE Trans. Image Process., vol. 9, no. 11, pp. 1877-1896, Nov. 2000.

[20] G. Harikumar and Y. Bresler, "Exact image deconvolution from multiple FIR blurs," IEEE Trans. Image Processing, vol. 8, no. 6, pp. 846-862, Jun. 1999

[21] H.-T. Pai and A. Bovik, "Exact multichannel blind image restoration," IEEE Signal Process. Lett., vol. 4, no. 8, pp. 217-220, Aug. 1997.

[22] — , "On eigenstructure-based direct multichannel blind image restoration," IEEE Trans. Image Process., vol. 10, no. 10, pp. 1434-1446, Oct. 2001.

[23] S. Pillai and B. Liang, "Blind image deconvolution using a robust GCD approach," IEEE Trans. Image Process., vol. 8, no. 2, pp. 295-301, Feb. 1999. 
[24] A. Rajagopalan and S. Chaudhuri, "A recursive algorithm for maximum likelihood-based identification of blur from multiple observations," IEEE Trans. Image Process., vol. 7, no. 7, pp. 1075-1079, Jul. 1998.

[25] M. Haindl and S. Šimberová, "Model-based restoration of short-exposure solar images," in Frontiers in Artificial Intelligence and Applications. ser. Knowledge-Based Intelligent Engeneering Systems, L. Jain and R. Howlett, Eds. Amsterdam, The Netherlands: IOS, 2002, vol. 87, pp. 697-706.

[26] G. Panci, P. Campisi, S. Colonnese, and G. Scarano, "Multichannel blind image deconvolution using the bussgang algorithm: Spatial and multiresolution approaches," IEEE Trans. Image Process., vol. 12, no. 11, pp. 1324-1337, Nov. 2003

[27] F. Sroubek and J. Flusser, "Multichannel blind iterative image restoration," IEEE Trans. Image Process., vol. 12, no. 9, pp. 1094-1106, Sep. 2003.

[28] B. Zitová and J. Flusser, "Image registration methods: A survey," Image Vis. Comput., vol. 21, pp. 977-1000, 2003.

[29] Z. Myles and N. V. Lobo, "Recovering affine motion and defocus blur simultaneously," IEEE Trans. Pattern Anal. Mach. Intell., vol. 20, no. 6, pp. 652-658, Jun. 1998.

[30] Y. Zhang, C. Wen, Y. Zhang, and Y. C. Soh, "Determination of blur and affine combined invariants by normalization," Pattern Recognit., vol. 35 , pp. 211-221, 2002.

[31] A. Kubota, K. Kodama, and K. Aizawa, "Registration and blur estimation methods for multiple differently focused images," in Proc. Int. Conf. Image Processing, vol. II, 1999, pp. 447-451.

[32] Z. Zhang and R. Blum, "A hybrid image registration technique for a digital camera image fusion application," Inf. Fusion, vol. 2, pp. 135-149, 2001.

[33] J. Flusser and T. Suk, "Degraded image analysis: An invariant approach," IEEE Trans. Pattern Anal. Mach. Intell., vol. 20, no. 6, pp. 590-603, Jun. 1998.

[34] J. Flusser, J. Boldyš, and B. Zitová, "Moment forms invariant to rotation and blur in arbitrary number of dimensions," IEEE Trans. Pattern Anal. Mach. Intell., vol. 25, no. 2, pp. 234-246, Feb. 2003.

[35] D. Mumford and J. Shah, "Optimal approximation by piecewise smooth functions and associated variational problems," Commun. Pure Appl. Math., vol. 42, pp. 577-685, 1989.

[36] G. Aubert and P. Kornprobst, Mathematical Problems in Image Processing. New York: Springer Verlag, 2002.

[37] A. Chambolle and P. Lions, "Image recovery via total variation minimization and related problems," Numer. Math., vol. 76, no. 2, pp. 167-188, Apr. 1997.
[38] D. Geman and G. Reynolds, "Constrained restoration and the recovery of discontinuities," IEEE Trans. Pattern Anal. Mach. Intell., vol. 14, no. 3, pp. 367-383, Mar. 1992.

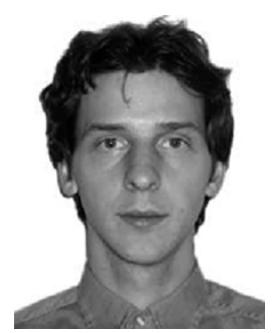

Filip Šroubek received the B.Sc. and M.Sc. degrees in computer science from the Czech Technical University, Prague, Czech Republic, in 1996 and 1998 , respectively, and the Ph.D. degree in computer science from the Charles University, Prague, in 2003.

Since 1999, he has been with the Institute of Information Theory and Automation, Academy of Sciences of the Czech Republic, Prague. Since 2000, he has been also with the Institute of Radiotechnique and Electronics, Academy of Sciences of the Czech Republic, Prague. His current research interests include all aspects of digital image processing and pattern recognition, particularly multichannel blind deconvolution, image denoising, image registration, and computer simulation and visualization of atomic collision processes.

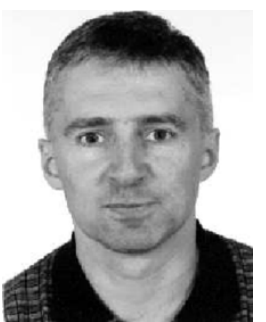

Jan Flusser (M'93-SM'03) received the M.Sc. degree in mathematical engineering from the Czech Technical University, Prague, Czech Republic, in 1985, and the Ph.D. degree in computer science and the D.Sc. degree in technical cybernetics from the Czechoslovak Academy of Sciences, Czech Republic, in 1990 and 2001, respectively.

Since 1985, he has been with the Institute of Information Theory and Automation, Academy of Sciences of the Czech Republic, Prague, where, since 1995, he has held the position of Head of the Department of Image Processing. Since 1991, he has been also affiliated with the Charles University, Prague, and the Czech Technical University, Prague, where he teaches courses on digital image processing and pattern recognition. His current research interests include all aspects of digital image processing and pattern recognition, namely 2-D object recognition, moment invariants, blind deconvolution, image registration, and image fusion, and he has authored or coauthored more than 80 research publications in these areas. 